\title{
Inhibition of respiratory syncytial virus infectivity by Ephedrae Herba and Cinnamomi Cortex through interaction with $\mathbf{G}$ glycoprotein
}

\section{Aya Fujikane}

Fukuoka University Hospital

\section{Atsuhiko Sakamoto}

Fukuoka University Hospital

Ryosuke Fujikane

Fukuoka Dental College

\section{Akinori Nishi}

Tsumura \& Co

\section{Yoshizumi Ishino}

Kyushu University

\section{Kenji Hiromatsu}

Fukuoka University

Shigeki Nabeshima ( $\nabla$ snabeshi@fukuoka-u.ac.jp )

Fukuoka University Hospital

\section{Article}

Keywords: respiratory syncytial virus (RSV), respiratory tract infection

Posted Date: March 9th, 2021

DOl: https://doi.org/10.21203/rs.3.rs-262616/v1

License: (9) This work is licensed under a Creative Commons Attribution 4.0 International License.

Read Full License

Version of Record: A version of this preprint was published at Communications Biology on January 25th, 2022. See the published version at https://doi.org/10.1038/s42003-022-03046-z. 


\section{Abstract}

Although respiratory syncytial virus (RSV) is a major cause of respiratory tract infection in children, no effective therapies are available. Recently, RSV G, the attachment glycoprotein, has become a major focus in the development of therapeutic strategies against RSV infection. Treatment of RSV-infected cultured cells with maoto, a traditional herbal medicine for acute febrile diseases, significantly reduced the viral RNA and titers. RSV attachment to the cell surface was inhibited both in the presence of maoto and when RSV particles were pre-treated with maoto. We demonstrated that maoto components, Ephedrae Herba (EH) and Cinnamomi Cortex (CC), specifically interacted with the central conserved domain (CCD) of $\mathrm{G}$ protein, and also found that this interaction blocked viral attachment to the cellular receptor CX3CR1. Genetic mutation of CX3C motif on the CCD, the epitope for CX3CR1, decreased the binding capacity to $\mathrm{EH}$ and $\mathrm{CC}$, suggesting that $\mathrm{CX} 3 \mathrm{C}$ motif was the target for $\mathrm{EH}$ and $\mathrm{CC}$. Finally, oral administration of maoto for five days to RSV-infected mice significantly reduced the lung viral titers. These experiments clearly showed the anti-RSV activity of EH and CC mixed in maoto. Taken together, this study provides new insights for the rational design of therapies against RSV infection.

\section{Introduction}

Respiratory syncytial virus (RSV) causes acute respiratory tract infection in individuals of all ages. Infection during infancy is especially common, and sometimes leads to hospitalization and death 1,2 . Despite decades of efforts, no effective therapies or vaccines are available ${ }^{3-5}$. Prophylaxis with anti-RSV antibodies, palivizumab, is limited to the high-risk children for preventing serious disease. At present, supportive care remains the only way for patients with RSV infection. Control of RSV infection is an urgent global problem, and the development of new antiviral drug is highly expected.

RSV belongs to the Orthopneumovirus genus of the Pneumoviridae family and has a negative-sense, single-stranded RNA genome. The viral envelope contains two major glycoproteins ${ }^{6}$ : the $\mathrm{G}$ glycoprotein, which mediates attachment to $\mathrm{CX} 3 \mathrm{C}$ chemokine receptor 1 (CX3CR1) on the cell surface ${ }^{7-10}$, and the $\mathrm{F}$ glycoprotein, which mediates fusion by interacting with the insulin-like growth factor 1 receptor and the coreceptor nucleolin ${ }^{11,12}$. The $G$ protein is more sequence diverse than $F$, except for a central conserved domain (CCD) that is nearly invariant across RSV strains ${ }^{13}$. Recently, the RSV G has become a major focus in the development of prophylactic and therapeutic strategies against RSV infection, with particular emphasis on the CCD because this domain contains the major epitope involved in cell surface attachment ${ }^{14,15}$. The CCD bears a conformationally constrained CX3C chemokine motif that is thought to be important for binding to CX3CR 1 and chemokine secretion ${ }^{16,17}$. Previous reports showed that RSV infection of mice or cultured lung cells could be inhibited by blockade of the CCD-CX3CR1 interaction using monoclonal antibodies against $\mathrm{G}$ protein or by genetic mutations of the CCD ${ }^{18-24}$.

Traditional herbal medicines have long played important roles in countries of the Far East, especially Japan, China, and Korea. Traditional herbal medicine, called Kampo, is accepted by the national medical 
insurance system of Japan, allowing the widespread use of these medicines by physicians. Maoto (mahuang-tang in Chinese) has shown clinical efficacy for treatment of common cold and influenza symptoms. Maoto is a multicomponent formulation extracted from four plants: Ephedrae Herba (EH), Cinnamomi Cortex (CC), Armeniacae Semen (AS), and Glycyrrhize Radix (GR) (Extended data Table 1). The formulation contains over 350 compounds; the major active compounds are ephedrine, methylephedrine, hippuric acid, and glycyrrhetinic acid ${ }^{25,26}$. We previously reported the results of two clinical trials showing that maoto was tolerable and effective in treating seasonal influenza by comparison with neuraminidase inhibitors ${ }^{27,28}$. Using our in vitro infection system, we previously showed that $\mathrm{EH}$ and $\mathrm{CC}$ in maoto caused influenza virus particles to remain in endosomes because of a failure in viral fusion with the endosome membrane through the elevation of endosomal $\mathrm{pH}$ condition ${ }^{29}$.

We report here a significant reduction of viral loads in RSV-infected A549 cells treated with maoto. We investigated the underlying mechanism and found that maoto components, especially $\mathrm{EH}$ and $\mathrm{CC}$, interacted with the CX3C chemokine motif on the CCD of RSV G protein and blocked attachment to cellular CX3CR1. These in vitro results were confirmed by in vivo analysis, that oral administration of maoto to mice infected with RSV showed the anti-viral and anti-inflammatory effect in lung. Taken together, the present study provides new insights into the treatment of RSV infection. Since maoto is a clinically proven drug, the further clinical application is feasible compared with newly designed antivirals which take high costs and limited administration routes.

\section{Results}

\section{Inhibitory effects of maoto in cultured cells infected with RSV}

We first assessed the antiviral activity of maoto in A549 cells infected with RSV (subtype A2) by real-time (RT)-PCR to quantitate intracellular viral RNA, as we did in previous experiments for influenza virus ${ }^{29}$. To avoid measuring progeny virus RNA, culture time was limited to 6 hours. Because the viral life cycle consists of attachment of virions to the cell surface followed by fusion, entry and subsequent replication, we divided the life cycle into two phases: the binding phase (first 1 hour post infection) and the entry/replication phase (subsequent 6-hour period after washing out residual virions) ${ }^{30}$. We found that viral RNA levels were remarkably decreased when maoto was present at the binding phase, whereas no significant decrease was observed when maoto was present at the entry/replication phase (Fig. 1a). When we pre-treated A549 cells with maoto prior to the binding phase, there was no decrease in viral RNA level (Fig. S1a), suggesting that host natural immunity or restriction factors with anti-RSV activity were not induced by maoto. On the basis of these results, in subsequent experiments maoto was added only at the binding phase. As shown in Fig. 1b, maoto treatment of A549 cells resulted in decreased RSV viral RNA levels in a dose-dependent manner. Together, these experiments suggested that maoto inhibits viral infectivity in the early phase of the viral life cycle.

A plaque-forming unit (PFU) assay showed significant reductions in the viral titres of culture supernatants treated with maoto at the binding phase. This result indicated that formation of infective progeny viruses 
was significantly decreased by maoto in a dose-dependent manner (Fig. 1c). We next visualized replicated viral components in host cells by immunofluorescence confocal microscopy. Translated viral G proteins (green fluorescence) were observed in the cytoplasm 24 hours post-RSV infection (Fig. S1b). The presence of maoto at the binding phase decreased the percentage of RSV-positive cells 24 hours postRSV infection in a dose-dependent manner (Fig. S1c).

Because maoto is composed of extracts from four plants (EH, AS, CC, and GR), we next assessed which plants were responsible for inhibition of viral infection. We found that EH and CC, but not AS and GR, showed significant antiviral effects (Fig. 1d). We next asked whether the antiviral effect of maoto was RSV-A2- or host cell-dependent. Treatment of RSV-B-infected A549 cells with maoto significantly reduced viral RNA levels in a dose-dependent manner (Fig. S2a). We also confirmed the anti-RSV-A effect of maoto in Vero cells derived from African green monkeys (Fig. S2b). These results indicated that maoto acts independently of both viral subtype and host cell line.

\section{Direct inhibitory effect of maoto on RSV}

Two mechanisms could explain the above results: either maoto interacted with RSV itself or with host cell receptors for RSV. To test potential direct effects of maoto on virions, high-concentration RSV was incubated with maoto for $0.5 \mathrm{~min}$ or $60 \mathrm{~min}$. A549 cells were infected with the treated virions (multiplicity of infection 1), then cultured for 6 hours (maoto concentration $<1 \mu \mathrm{g} / \mathrm{mL}$ ) (Fig. 2a). We observed a significant decrease in viral RNA level when virions were exposed to maoto for 60 min, suggesting a direct effect of maoto on RSV. To exclude potential effects of maoto on cellular receptors, cells were incubated with maoto for 1 hour on ice prior to RSV infection. No decrease in viral RNA level was observed, suggesting that maoto had no effect on cellular receptors for RSV (Fig. S3). Therefore, we next asked whether maoto disrupted RSV or interacted with specific RSV epitopes to prevent binding to cells. To distinguish between these possibilities, RSV attached to the cell surface was treated with maoto on ice. At low temperatures, virions remain bound to cellular receptors without entering the cytoplasm because cellular metabolism is slowed ${ }^{30}$. Following maoto treatment, cells were cultivated at $37^{\circ} \mathrm{C}$ for 24 hours without maoto. As shown in Fig. 2b, viral RNA was propagated in spite of prior exposure to maoto, indicating that maoto does not directly inactivate RSV but may instead interact with RSV epitopes required for cellular receptor attachment.

To investigate whether maoto could inhibit viral attachment to the cell surface, we further examined the amount of viral RNA and protein located on the cell surface at the binding phase. RSV was allowed to bind to cells in the presence or absence of maoto for 1 hour, and then the amount of viral RNA in cell extracts was assayed by RT-PCR (Fig. 2c). Cell surface viral RNA levels were significantly reduced by maoto treatment. This result was confirmed at the protein level: cell surface RSV G protein levels were reduced with increasing maoto concentration as shown by western blotting (Fig. 2d). RSV attachment to the cell surface was observed and quantified by immunofluorescence confocal microscopy (Fig. 2e). Virion foci significantly decreased in a dose-dependent manner when cells were treated with maoto (Fig. S4). 


\section{Interaction of maoto components with RSV G protein}

We hypothesized that maoto components might interact with viral envelope glycoproteins to inhibit RSV attachment to cellular receptors. We focused on the RSV G protein, which was reported to be the major protein responsible for virion attachment to host cell receptors. We examined the binding affinity between maoto and RSV G protein using surface plasmon resonance (SPR). When we injected various concentrations of maoto over $\mathrm{G}$ protein-immobilized surfaces, dose-dependent binding was observed (Fig. 3a). After washing (> 120 seconds), a significant proportion of the injected maoto components remained on $\mathrm{G}$ protein-immobilized surfaces, indicating that the binding affinity between maoto and RSV $\mathrm{G}$ protein was strong. In contrast, maoto did not interact with severe acute respiratory syndrome coronavirus-2 S glycoprotein (Fig. 3b), suggesting that binding of maoto to RSV G protein was specific. Because maoto is composed of crude extracts whose components have varying molecular weights, dissociation constants (KD) could not be calculated. We next assessed which maoto components (EH, $\mathrm{CC}, \mathrm{GR}$ or AS) interacted with RSV G protein. We found that EH and CC, but not GR and AS, bound RSV G protein (Fig. 3c). This result was consistent with the effects of these components on cultured cells (Fig. 1d). Because the RSV G protein consists of heavily glycosylated mucin-like domains, it shows a broad band in western blotting studies (Fig. 3d, left). We next examined whether maoto targeted proteins or glycans of the RSV G protein. We prepared a non-glycosylated RSV G protein extracellular domain in Escherichia coli (Fig. 3d, right). Dose-dependent binding of maoto to non-glycosylated G protein was observed by SPR (Fig. 3e), suggesting that the maoto reaction with RSV G protein was not glycandependent.

We next examined whether the maoto-RSV G protein interaction inhibited the attachment of RSV to cells. As shown in Fig. 3f, CX3CR1, the target for RSV-G attachment, was present on A549 cells as shown by immunofluorescence confocal microscopy using a monoclonal anti-CX3CR1 antibody. We investigated the effect of maoto on the interaction between RSV G protein and cellular CX3CR1 using a proximity ligation assay (PLA) which was developed for the detection of protein-protein interaction in situ. RSV was allowed to attach to A549 cells on ice in the presence or absence of maoto, followed by the PLA (Fig. 3g). Many foci reflecting the proximal association of RSV G protein and CX3CR1 were observed on A549 cells in the absence of maoto. However, significantly decreased numbers of foci were observed in cells treated with maoto (Fig. S5). These results demonstrated that specific binding of G protein to CX3XR1 was inhibited by maoto.

\section{Specific interaction of maoto with the CX3C motif on CCD}

The CX3C motif on the CCD of RSV G protein was reported to be a candidate epitope responsible for virion attachment to $\mathrm{CX} 3 \mathrm{CR} 1^{17}$. Therefore, we next examined whether maoto could interact directly with the CCD CX3C motif. We synthesized a peptide derived from the CCD (CX3C, aa 164-186) and a mutant peptide in the CX3C motif (SX3S), the later was replaced cysteines of CX3C motif into serines (Fig. 4a) ${ }^{9}$. SPR revealed strong dose-dependent binding of maoto to the CX3C peptide (Fig. $4 \mathrm{~b}$ ), but around $50 \%$ weaker binding to the SX3S peptide (Fig. 4c). Both EH and CC bound to the two peptides similarly to 
maoto (Fig. $4 \mathrm{~d}$ and e). These results demonstrated that maoto and its components (EH and CC) preferentially bind the CX3C motif on the RSV G protein CCD to inhibit virion attachment to CX3CR1.

The chemokine domain of CX3CL1 (known as fractalkine) bears a CX3C motif (Fig. 5a) responsible for stimulating cytokine production following interaction with CX3CR $1^{31}$. If maoto components interacted with the CX3C motif of fractalkine, maoto might also inhibit the attachment of fractalkine to CX3XR1. Because A549 cells do not produce interleukin (IL)- 6 in response to fractalkine alone, cells were first primed with phorbol 12-myristate 13-acetate (PMA) and ionomycin, then incubated with fractalkine for 20 hours. Intracellular IL-6 mRNA levels were significantly increased in A549 cells treated with fractalkine, but no increase was observed when maoto was added simultaneously (Fig. 5b). The interaction between the CX3C motif of fractalkine and maoto was confirmed by SPR (Fig. 5c). These results further suggested that binding of maoto to the CX3C motif of RSV G protein mediates anti-RSV activity.

\section{Rreduces viral loads by maoto in mice infected with RSV}

To confirm the antiviral effect of maoto in vivo, mice were intranasally infected with RSV and orally administered maoto, water, or prednisolone for 5 days. No mice died following experimental infection. Bronchoalveolar lavage fluid (BALF) was collected 5 days post-inoculation, and viral loads were examined by PFU assay (Fig. 6a). Viral titres were remarkably reduced in maoto-treated mice compared with water- and prednisolone-treated control mice. Histopathological analysis of the lungs of water- and prednisolone-treated mice showed infiltration of mononuclear cells in the interstitial and perivascular regions (Fig. 6b). In contrast, lung sections from maoto-treated mice revealed less infiltration of inflammatory cells. Because RSV induces inflammatory responses in the lung partly via the chemokine receptor, CX3CR1, we assessed levels of the inflammatory cytokines, IL-1 $\beta$ and IL-6, in the murine lung. Lung IL-1 $\beta$ and IL-6 mRNA levels in maoto-treated mice were slightly but not significantly decreased (Figs. S3a and $b$ ). These results suggested that lung injury following RSV infection may be reduced by administration of maoto. Thus, the antiviral activity of maoto in vitro was confirmed by the in vivo experiments.

\section{Discussion}

RSV infection in children has become a global issue for its high morbidity and mortality. The present study, showing that the traditional herbal medicine maoto has an antiviral activity against RSV, provides new insights for the rational design of therapies against RSV infection. Maoto is a widely distributed drug in Japan, and its tolerability and effectiveness in patients with acute respiratory infections are widely accepted. Although maoto is composed of crude extracts, the clinical product is supplied as powders or granules, making it easy to take orally. Guidelines for quality management of the constitutive herbs of maoto are defined in the Japanese Pharmacopeia. Furthermore, the low cost of maoto provides an economic benefit for developing countries compared with antiviral monoclonal antibodies, which are costly and limited to intravenous administration. Therefore, repositioning and clinical trials of maoto may be more feasible than developing other candidate drugs to treat RSV infection. We are currently designing a clinical protocol for treatment of RSV infection in children using maoto granules. 
The epitope in RSV G protein, CCD, responsible for attachment to host cells has recently been targeted by therapeutic and prophylactic monoclonal antibodies. However, poor antigenicity of the CCD and high development costs have made it difficult to develop effective neutralizing antibodies for clinical use. Both maoto and novel antibodies against the CCD may inhibit the infectivity and inflammatory activity of RSV. They were thoght to block attachment of CCD CX3C motif to CX3CR1. At present, it is unclear whether EH and $C C$ in maoto interact preferentially with the CCD CX3C motif or with broader sites including the CX3C motif. It is also possible that maoto may interact with the RSV F protein or the heparin-binding domain immediately adjacent to the $\mathrm{CX} 3 \mathrm{C}$ motif of CCD; the former fuses to host cell membrane for uncoating and the latter plays an important role in attachment to some kinds of cultured cells ${ }^{32}$. Our preliminary experiments by SPR showed that $\mathrm{EH}$ and $\mathrm{CC}$ could also interact with $\mathrm{F}$ protein. If maoto can interact with multiple viral components, it would broaden the spectrum of targets acted on by maoto to inhibit RSV attachment, and it may help prevent drug resistance. The anti-RSV effect of maoto was mediated by $\mathrm{EH}$ and CC, each containing hundreds of pharmacologically active compounds, and it remains possible that multiple compounds interact with the RSV G protein CCD. We are working to identify the specific molecule(s) involved in the antiviral effect of EH and CC by liquid chromatography and mass spectrometry. Preliminal experiments suggest that $\mathrm{EH}$ has at least two fractions identified by liquid chromatography to specifically inhibit the infectivity of RSV. According to the literatures, other natural compounds derived from plants were reported to have an anti-RSV activity ${ }^{33-36}$. As well as maoto, Baicalin from Scutellaria Baicalensis blocked RSV attachment, however the precise mechanisms in molecular levels are not elucidated ${ }^{30}$.

The unique characteristic of traditional herbal medicine, called kampo, was marked by the multiple components with diverse functions. Maoto may include molecules for relieving symptoms as well as those for inhibiting virus infection. Of plants mixed in maoto, EH and AS had the bronchodilation capacity which is important for RSV-infected children with bronchial asthma ${ }^{37}$. We have recently reported that maoto had a clinical effectiveness for seasonal influenza without inferiority to standard neuraminidase inhibitors. The anti-viral mechanism against influenza is that $\mathrm{EH}$ and $\mathrm{CC}$ in maoto inhibited the endosomal acidification, resulting in the inhibition of virus uncoating ${ }^{29}$. Interestingly, this mechanism is entirely different from the anti-viral mechanism against RSV infection in the present study. The effective concentration of maoto in the culture system of the present study was ten times less than that of influenza study. However, we also found that increasing dose of maoto could inhibit the propagation of RSV RNA even in the entry or post-uncoating phase (Fig. S7), suggesting that host defense mechanisms or another antiviral mechanisms were induced when cells were treated with high concentration of maoto. Unlike the existing anti-virals, there may be several independent anti-viral mechanisms in maoto. We have already reported that maoto could inhibit the serum proinflammatory cytokine responses and prostaglandin E2 levels in rats after Poly I:C injection, which was the model of acute RNA virus infection 25. Taken together, it is suggested that maoto has both anti-viral and anti-inflammatory activities.

Maoto is the first clinical drug targeting the CCD of RSV G protein other than monoclonal antibodies ${ }^{5}$. The mechanism is that $\mathrm{EH}$ and $\mathrm{CC}$ interact with $\mathrm{CX} 3 \mathrm{C}$ motif on $\mathrm{G}$ protein $\mathrm{CCD}$ to block attachment to 
CX3CR1, the receptor for RSV G protein. The anti-viral effect of maoto was confirmed by the mouse infection model. The benefits of maoto are its low cost, ease of administration, and clinical track record in Japan. Considering the number of children suffering from RSV infection in the world, drug repositioning and clinical trials of maoto are justified.

\section{Materials And Methods}

\section{Cells and Viruses}

A549 cell line derived from human lung cancer, and RSV-A and -B were purchased from ATCC (Manassas, Virginia, US). Vero cells were from lab stock. The cell lines were cultivated in D-MEM supplemented with $10 \% \mathrm{FBS}$ and penicillin/streptomycin at $37^{\circ} \mathrm{C}$ in $5 \% \mathrm{CO}_{2}$. Viruses were initially propagated in $\mathrm{A} 549$ cells. The supernatant of culture was titered by PFU assay, and stored frozen under $-80^{\circ} \mathrm{C}$, as a stock. Titers of viruses from culture supernatants and bronchoalveolar lavage fluid (BALF) from lungs of RSV-infected mice were determined by conventional PFU assay ${ }^{38}$. Briefly, HEp-2 cells were inoculated with RSV in serially diluted medium, followed by the incubation for $60 \mathrm{~min}$, and then, overlaid with $0.3 \%$ warm Noble agar (Sigma-Aldrich, St. Louis, Missouri, US). HEp-2 cells were cultured for 6 days at $37^{\circ} \mathrm{C}$ in $5 \% \mathrm{CO}_{2}$ condition. The cells were stained with neutral red and viral titers were determined by counting plaques.

\section{Antiviral Reagents}

Maoto, provided by Tsumura (Tokyo, Japan), is an extracted mixture of four plants (Table S1), Ephedrae Herba (ephedra; EH), Armeniacae Semen (apricot; AS), Cinnamomi Cortex (cinnamon; CC), and Glycyrrhize Radix (licorice; GR). Crude drug pieces from EH, AS, CC, and GR were decocted by boiling water at a ratio of 10:10:8:3, respectively. Maoto powder was obtained via concentration and spray-drying of the decoction. The quality management of maoto is comformed to the Japanese Pharmacopeia. Before experimental use, maoto powder was dissolved and incubated in warm PBS for 1 hour, and supernatant was collected after sedimentation at $3,000 \mathrm{xg}$, then filtered through a $0.45 \mu \mathrm{m}$ membrane filter. Dissolved maoto were stored at $-80^{\circ} \mathrm{C}$ until use. As reported in the previous study, cytotoxicity of maoto on A549 cells was seen over $1,000 \mu \mathrm{g} / \mathrm{ml}$ for 24 hours.

\section{Real-time PCR analysis}

RNA extraction was carried out using ISOGEN II (Nippon Gene, Tokyo, Japan) and cDNAs were synthesized with a prime script RT reagent kit with gDNA eraser (Toyobo, Osaka, Japan), according to the manufacturer's instructions.

Real-time PCR analysis was performed using CFX connect real-time PCR detection system (Bio-Rad; Hercules, California, US) with SYBR Green Kit (Takara, Kusatsu, Shiga, Japan). The sequences of primers are listed below (forward and revers):

human GAPDH: GCACCGTCAAGGCTGAGAAC and ATGGTGGTGAAGACGCCAGT

RSV-A (N region): CATCCAGCAAATACACCATCCA and TTCTGCACATCATAATTAGGAGTATCAA 
RSV-B (P region): ACGCTACAAGGGCCTCATAC and TGCAATGCCAAAGTGCACAA

mouse IL-1ß: CCTTCCAGGATGAGGACATGA and TGAGTCACAGAGGATGGGCTC

mouse IL-6: GAGGATACCACTCCCAACAGACC and AAGTGCATCATCGTTGTTCATACA

human IL-6: ACTCACCTCTTCAGAACGAATTG and CCATCTTTGGAAGGTTCAGGTTG Western blotting analysis

Cells were harvested in a lysis buffer (100 mM Tris-HCl (pH 6.8), 2\% SDS, 20\% glycerol, $2 \% \beta$ mercaptoethanol, and $0.4 \mathrm{mg} / \mathrm{ml}$ bromophenol blue), and boiled at $100^{\circ} \mathrm{C}$ for 10 minutes. Soluble proteins were separated by SDS-PAGE and transferred to a PVDF membrane (Bio-Rad) by Trans-blot turbo (Bio-Rad). The membrane was immersed with the Can Get Signal PVDF blocking Reagent (Toyobo) for 1 h, and incubated with monoclonal antibodies against RSV-G protein (94966; Abcam, Cambridge, UK) and B-actin (Sigma-Aldrich, St. Louis, Missouri, US) for overnight. After washing with PBS containing $0.1 \%$ tween 20 , the membrane was blotted with horseradish peroxidase-conjugated secondary antibody followed by visualization with a chemiluminescence agent, ECL Prime Western Blotting Detection Reagent (GE Healthcare, Chicago, Illinois, US) by LAS-3000 (GE Healthcare).

\section{Immunofluorescence confocal microscopy}

Cells were fixed with $4 \%$ paraformaldehyde at room temperatures for 15 minutes, and stained with primary antibody against RSV G protein and CXCR1 (8021; Abcam), followed by further incubation with secondary Ab conjugated with Alexa Fluor 488 (ThermoFisher, Waltham, Massachusetts, US). Samples were mounted by mounting agent (Prolong Diamond antifade mountant with DAPI; ThermoFisher) and observed under a confocal laser scanning microscopy LSM710 (Zeiss, Oberkochen, Germany). In some experiment (figs. S4 and 5), fluorescein foci were counted by ImageJ software (Wayne Rasband, Bethesda, Maryland, US).

\section{Surface plasmon resonance (SPR)}

A Biacore $J$ system (GE Healthcare) was used to study the physical interaction between $\mathrm{G}$ protein, CCD peptide, and fractalkine with maoto components. All experiments were conducted at $25^{\circ} \mathrm{C}$. Purified $\mathrm{G}$ protein (with sugar or without sugar) and CCD peptide from RSV strain A containing a C-terminal 6xHis tag were immobilized on a Ni-NTA sensor chip (GE Healthcare). Fractalkine was immobilized on a CM5 sensor chip (GE Healthcare). Samples of maoto were injected at a flow rate of $30 \mu \mathrm{l} / \mathrm{min}$ for association. Disassociation was performed over 120 seconds interval. The apparent affinity was evaluated with BIAevaluation software (GE Healthcare).

\section{Proximity ligation assay (PLA)}

PLA (Duolink; Sigma-Aldrich) was done according to the manufacture's instruction. Briefly, A549 cells were attached with RSV for one hour on ice, and washed with cold PBS. The cells were fixed with $4 \%$ paraformaldehyde at room temperature for $15 \mathrm{~min}$, followed by incubation with the blocking agent. The samples were incubated with primary antibodies against $\mathrm{G}$ protein (Abcam) and CX3CR1 (Abcam), 
followed by binding of the PLA probes. Circle DNA between the two probes was amplified with fluorescent-labeled oligonucleotides. The samples were analyzed by the confocal microscopy, LSM-710.

\section{Production of recombinant protein and peptide}

Glycosylated RSV G was purchased from (Sino Biological, Beijing, China). Non-glycosylated recombinant RSV $G$ was produced as described previously with modification ${ }^{39}$. E. coli BL21(DE3) cells were transformed with a gene encoding RSV G extracellular domain (aa 66-297) with His-tag at C-terminus in $\mathrm{pET} 21 \mathrm{a}(+)$ vector and cultivated at $37^{\circ} \mathrm{C}$ until OD600 reached to 0.3 . Then, isopropyl $\beta-D-$ thiogalactopyranoside was added and the cells were further cultivated for $6 \mathrm{~h}$. The cells were harvested and resuspended in the lysis buffer and sonicated. After removal of cell debris, the proteins were precipitated with the saturated ammonium sulfate at $4^{\circ} \mathrm{C}$. The precipitant was resuspended in a high salt buffer (10 mM Tris- $\mathrm{HCl}, \mathrm{pH} 8.0,1 \mathrm{M}$ ammonium sulfate) and subjected to an hydrophobic column (HitrapPhenyl HP column, GE Healthcare) connecting to FPLC (AKTA Explorer 10, GE Healthcare). The proteins were eluted with $10 \mathrm{mM}$ Tris- $\mathrm{HCl} \mathrm{pH} \mathrm{8.0,} \mathrm{the} \mathrm{eluate} \mathrm{was} \mathrm{diluted} \mathrm{with} \mathrm{a} \mathrm{buffer}(20 \mathrm{mM}$ Tris HCl, $\mathrm{pH} 7.6$ and $0.5 \mathrm{M} \mathrm{NaCl}$ ) and applied to an affinity column (His-trap HP column, GE Healthcare) and bound proteins were eluted with a linear gradient $(20 \mathrm{mM}-500 \mathrm{mM}$ imidazole) of the buffer. Purified fractions were confirmed by $15 \%$ SDS-PAGE followed by CBB staining, and stored at $4{ }^{\circ} \mathrm{C}$. The CCD peptides with or without CX3C motif (aa 164-186) with 6xHis-tag at C-terminus were purchased from Cosmo Bio, and their sequences of amino acid residues are listed below.

CCD with CX3C motif: HFEVFNFVPCSICSNNPTCWAICHHHHHH

CCD without CX3C motif: HFEVFNFVPCSICSNNPTSWAISHHHHHH

\section{Stimulation of A549 cells by fractalkine}

A549 cells were stimulated with $50 \mathrm{ng} / \mathrm{ml}$ PMA (Sigma-Aldrich) and $1 \mu \mathrm{g} / \mathrm{ml}$ ionomycin (Sigma-Aldrich) for 4 hours, washed, and followed by treatment with $100 \mathrm{nM}$ fractalkine (Fujifilm, Tkyo, Japan) in the presence or absence of maoto $(25 \mu \mathrm{g} / \mathrm{ml})$ for 20 hours. Cell lysates were assayed by RT-PCR for IL-6 mRNA.

\section{Experimental murine infection with RSV}

Female BALB/c mice were purchased from SLC (Hamamatsu, Shizuoka, Japan), and used for the experiment at 6 weeks old. For the RSV infection, RSV $\left(1.8 \times 10^{6} \mathrm{PFU} / 0.1 \mathrm{ml} / \mathrm{mouse}\right)$ was intranasally inoculated. Maoto (1 or $2 \mathrm{~g} / 10 \mathrm{ml} / \mathrm{kg}$ ) ( $\mathrm{N}=8$ in each group), prednisolone $(10 \mathrm{mg} / 10 \mathrm{ml} / \mathrm{kg})(\mathrm{N}=6)$ or distilled water $(10 \mathrm{ml} / \mathrm{kg})(\mathrm{N}=8)$ were orally administrated for 5 days from one hour after RSV inoculation until 4 days post-inoculation. Mice were sacrificed at 5 days post-inoculation, and collected BALF and lung tissue. For BALF sampling, $0.5 \mathrm{ml}$ saline were injected three times into the trachea and retrieved fluids. The left lung tissue was kept frozen in RNAlater (Invitrogen, Carlsbad, California, US) and other left lung was fixed with $10 \%$ formaldehyde solution. Paraffin sections of fixed tissue specimens were stained with hematoxylin and eosin. IL-1 $\beta$ and IL- 6 mRNA from 3 individual mice were evaluated by RT-PCR. All animal experiments were approved by the Laboratory Animal Committee of Nihon Bioresearch (Hashima, 
Gifu, Japan) and Tsumura \& Co (Tokyo, Japan), and performed in accordance with guidelines for the conduct of animal experiments in ministry of health, labour and welfare, Japan.

\section{Statistical analysis}

All the experimental data except for murine infection model were obtained from at least three independent experiments. Results were shown as means \pm standard deviation (errorbars). The statistical significances of group differences were determined by paired or unpaired Student's $t$ test. If the parameters were not distributed normally, they were determined by Mann-Whitney $U$ test. $P$ values less than 0.05 were considered significant. Data were analyzed with GraphPad Prism software (San Diego, California, US).

\section{Declarations}

Acknowledgments: We thank N. Okabe from the Life Science and Environment Research Centre for his valuable advice and S. Ishino from Kyushu University for help with SPR experiments. We also thank K. Ishii, R. Itoh, B. Chou, Y. Kurihara, and A. Shimizu from Fukuoka University for technical advice. The structural model of CCD was generated using Phyre2 software (http://www.sbg.bio.ic.ac.uk/phyre2/html/page.cgi?id=help). The animal experiment was conducted by Nihon Bioresearch, Inc. This work was supported in part by a grant-in-aid for scientific research from the Japan Society for the Promotion of Science (19K07881).

Author contributions: A.F. performed all aspects of this study. A.S. helped cell cultures and production of proteins. R.F. performed confocal microscopy, PLA experiments, and helped production of recombinant proteins. A.N. planned and conducted mouse experiments, and provided reagents. Y.I. helped to perform SPR experiments. K.H. helped to conceive the study and provided expertise. S.N. conceived and coordinated the study, and helped to write the manuscript. All authors read and approved final manuscript.

Competing interest: A.N is employed by Tsumura \& Co. S.N has financial interests in Tsumura \& Co. relevant to this research. The other authors declare no competing financial interests.

\section{References}

1 Shi, T. et al. Global, regional, and national disease burden estimates of acute lower respiratory infections due to respiratory syncytial virus in young children in 2015: a systematic review and modelling study. Lancet 390, 946-958, doi:10.1016/S0140-6736(17)30938-8 (2017).

2 Simões, E. A. F. et al. Past, Present and Future Approaches to the Prevention and Treatment of Respiratory Syncytial Virus Infection in Children. Infect Dis Ther 7, 87-120, doi:10.1007/s40121-018-0188z (2018). 
3 Barr, R., Green, C. A., Sande, C. J. \& Drysdale, S. B. Respiratory syncytial virus: diagnosis, prevention and management. Ther Adv Infect Dis 6, 2049936119865798, doi:10.1177/2049936119865798 (2019).

$4 \quad$ Beigel, J. H. et al. Advances in respiratory virus therapeutics - A meeting report from the 6th isirv Antiviral Group conference. Antiviral Res 167, 45-67, doi:10.1016/j.antiviral.2019.04.006 (2019).

$5 \quad$ Heylen, E., Neyts, J. \& Jochmans, D. Drug candidates and model systems in respiratory syncytial virus antiviral drug discovery. Biochem Pharmaco/ 127, 1-12, doi:10.1016/j.bcp.2016.09.014 (2017).

6 McLellan, J. S., Ray, W. C. \& Peeples, M. E. Structure and function of respiratory syncytial virus surface glycoproteins. Curr Top Microbiol Immuno/ 372, 83-104, doi:10.1007/978-3-642-38919-1_4 (2013).

7 Anderson, C. S. et al. CX3CR1 as a respiratory syncytial virus receptor in pediatric human lung. Pediatr Res 87, 862-867, doi:10.1038/s41390-019-0677-0 (2020).

8 Chirkova, T. et al. CX3CR1 is an important surface molecule for respiratory syncytial virus infection in human airway epithelial cells. J Gen Viro/ 96, 2543-2556, doi:10.1099/vir.0.000218 (2015).

9 Johnson, S. M. et al. Respiratory Syncytial Virus Uses CX3CR1 as a Receptor on Primary Human Airway Epithelial Cultures. PLoS Pathog 11, e1005318, doi:10.1371/journal.ppat.1005318 (2015).

10 Langedijk, J. P., Schaaper, W. M., Meloen, R. H. \& van Oirschot, J. T. Proposed three-dimensional model for the attachment protein G of respiratory syncytial virus. J Gen Viro/ 77 (Pt 6), 1249-1257, doi:10.1099/0022-1317-77-6-1249 (1996).

11 Griffiths, C. D. et al. IGF1R is an entry receptor for respiratory syncytial virus. Nature, doi:10.1038/s41586-020-2369-7 (2020).

12 Tayyari, F. et al. Identification of nucleolin as a cellular receptor for human respiratory syncytial virus. Nat Med 17, 1132-1135, doi:10.1038/nm.2444 (2011).

13 Plotnicky-Gilquin, H. et al. Identification of multiple protective epitopes (protectopes) in the central conserved domain of a prototype human respiratory syncytial virus $\mathrm{G}$ protein. $J$ Viro/ 73, 56375645 (1999).

14 Battles, M. B. \& McLellan, J. S. Respiratory syncytial virus entry and how to block it. Nat Rev Microbio/ 17, 233-245, doi:10.1038/s41579-019-0149-x (2019).

15 Tripp, R. A., Power, U. F., Openshaw, P. J. M. \& Kauvar, L. M. Respiratory Syncytial Virus: Targeting the G Protein Provides a New Approach for an Old Problem. J Viro/ 92, doi:10.1128/JVI.01302-17 (2018). 
16 Arnold, R., König, B., Werchau, H. \& König, W. Respiratory syncytial virus deficient in soluble G protein induced an increased proinflammatory response in human lung epithelial cells. Virology 330, 384397, doi:10.1016/j.virol.2004.10.004 (2004).

17 Tripp, R. A. et al. CX3C chemokine mimicry by respiratory syncytial virus $\mathrm{G}$ glycoprotein. Nat Immuno/ 2, 732-738, doi:10.1038/90675 (2001).

18 Boyoglu-Barnum, S. et al. Mutating the CX3C Motif in the G Protein Should Make a Live Respiratory Syncytial Virus Vaccine Safer and More Effective. J Viro/ 91, doi:10.1128/JVI.02059-16 (2017).

19 Caidi, H. et al. Anti-respiratory syncytial virus (RSV) G monoclonal antibodies reduce lung inflammation and viral lung titers when delivered therapeutically in a BALB/c mouse model. Antiviral Res 154, 149-157, doi:10.1016/j.antiviral.2018.04.014 (2018).

20 Cortjens, B. et al. Broadly Reactive Anti-Respiratory Syncytial Virus G Antibodies from Exposed Individuals Effectively Inhibit Infection of Primary Airway Epithelial Cells. J Viro/ 91, doi:10.1128/JVI.02357-16 (2017).

21 Fedechkin, S. O., George, N. L., Wolff, J. T., Kauvar, L. M. \& DuBois, R. M. Structures of respiratory syncytial virus $\mathrm{G}$ antigen bound to broadly neutralizing antibodies. Sci Immuno/3, doi:10.1126/sciimmunol.aar3534 (2018).

22 Ha, B. et al. Mutation of Respiratory Syncytial Virus G Protein's CX3C Motif Attenuates Infection in Cotton Rats and Primary Human Airway Epithelial Cells. Vaccines (Basel) 7, doi:10.3390/vaccines7030069 (2019).

23 Jones, H. G. et al. Structural basis for recognition of the central conserved region of RSV G by neutralizing human antibodies. PLoS Pathog 14, e1006935, doi:10.1371/journal.ppat.1006935 (2018).

24 Lee, H. J., Lee, J. Y., Park, M. H., Kim, J. Y. \& Chang, J. Monoclonal Antibody against G Glycoprotein Increases Respiratory Syncytial Virus Clearance In Vivo and Prevents Vaccine-Enhanced Diseases. PLoS One 12, e0169139, doi:10.1371/journal.pone.0169139 (2017).

25 Nishi, A. et al. Deconstructing the traditional Japanese medicine "Kampo": compounds, metabolites and pharmacological profile of maoto, a remedy for flu-like symptoms. NPJ Syst Biol App/3, 32, doi:10.1038/s41540-017-0032-1 (2017).

26 Ohbuchi, K. et al. Differential annotation of converted metabolites (DAC-Met): Exploration of Maoto (Ma-huang-tang)-derived metabolites in plasma using high-resolution mass spectrometry. Metabolomics 16, 63, doi:10.1007/s11306-020-01681-3 (2020).

27 Nabeshima, S. et al. A comparison of oseltamivir with moat, a traditional herbal medicine, for the treatment of adult seasonal influenza A. J Trad Med 27, 148-156 (2010). 
28 Nabeshima, S. et al. A randomized, controlled trial comparing traditional herbal medicine and neuraminidase inhibitors in the treatment of seasonal influenza. $J$ Infect Chemother 18, 534-543, doi:10.1007/s10156-012-0378-7 (2012).

29 Masui, S. et al. Maoto, a Traditional Japanese Herbal Medicine, Inhibits Uncoating of Influenza Virus. Evid Based Complement Alternat Med 2017, 1062065, doi:10.1155/2017/1062065 (2017).

30 Shi, H. et al. Baicalin from Scutellaria baicalensis blocks respiratory syncytial virus (RSV) infection and reduces inflammatory cell infiltration and lung injury in mice. Sci Rep 6, 35851, doi:10.1038/srep35851 (2016).

31 Imai, T. et al. Identification and molecular characterization of fractalkine receptor CX3CR1, which mediates both leukocyte migration and adhesion. Cel/ 91, 521-530, doi:10.1016/s0092-8674(00)80438-9 (1997).

32 Feldman, S. A., Hendry, R. M. \& Beeler, J. A. Identification of a linear heparin binding domain for human respiratory syncytial virus attachment glycoprotein G. J Virol 73, 6610-6617 (1999).

33 Hou, S., Xu, X., Wang, Y. \& Yang, Y. Ephedrannin B exerts anti-viral and anti-inflammatory properties in BEAS-2B cells infected with respiratory syncytial virus. J Biosci 45 (2020).

34 Lee, J. W. et al. Grape Seed Proanthocyanidin Inhibits Mucin Synthesis and Viral Replication by Suppression of AP-1 and NF-KB via p38 MAPKs/JNK Signaling Pathways in Respiratory Syncytial VirusInfected A549 Cells. J Agric Food Chem 65, 4472-4483, doi:10.1021/acs.jafc.7b00923 (2017).

35 Machado, V. B. et al. Biophysical and flavonoid-binding studies of the G protein ectodomain of group A human respiratory syncytial virus. Heliyon 5, e01394, doi:10.1016/j.heliyon.2019.e01394 (2019).

36 Tang, W. et al. Small molecule inhibits respiratory syncytial virus entry and infection by blocking the interaction of the viral fusion protein with the cell membrane. FASEB J 33, 4287-4299, doi:10.1096/fj.201800579R (2019).

37 Huang, T. P. et al. Characteristics of traditional Chinese medicine use in children with asthma: a nationwide population-based study. Allergy 68, 1610-1613, doi:10.1111/all.12273 (2013).

38 McKimm-Breschkin, J. L. A simplified plaque assay for respiratory syncytial virus--direct visualization of plaques without immunostaining. J Virol Methods 120, 113-117, doi:10.1016/j.jviromet.2004.02.020 (2004).

39 Fuentes, S., Coyle, E. M., Golding, H. \& Khurana, S. Nonglycosylated G-Protein Vaccine Protects against Homologous and Heterologous Respiratory Syncytial Virus (RSV) Challenge, while Glycosylated G Enhances RSV Lung Pathology and Cytokine Levels. J Viro/ 89, 8193-8205, doi:10.1128/JVI.00133-15 (2015). 
Figures

a
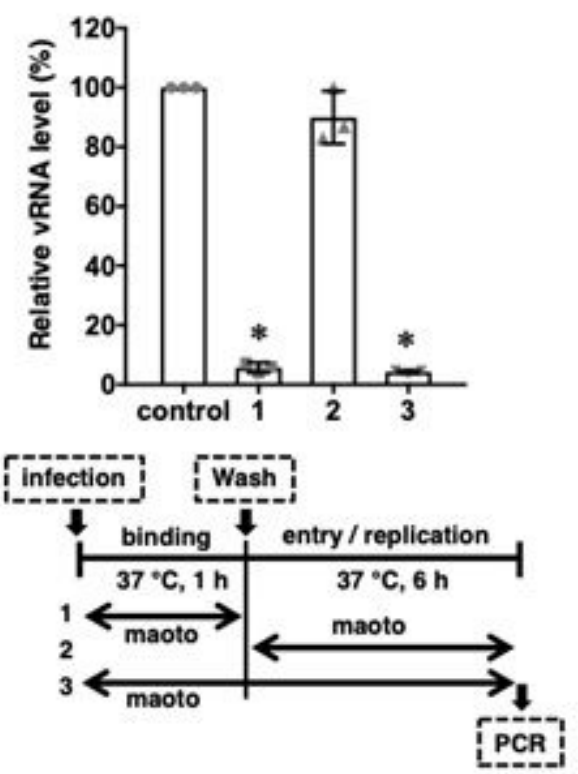

C
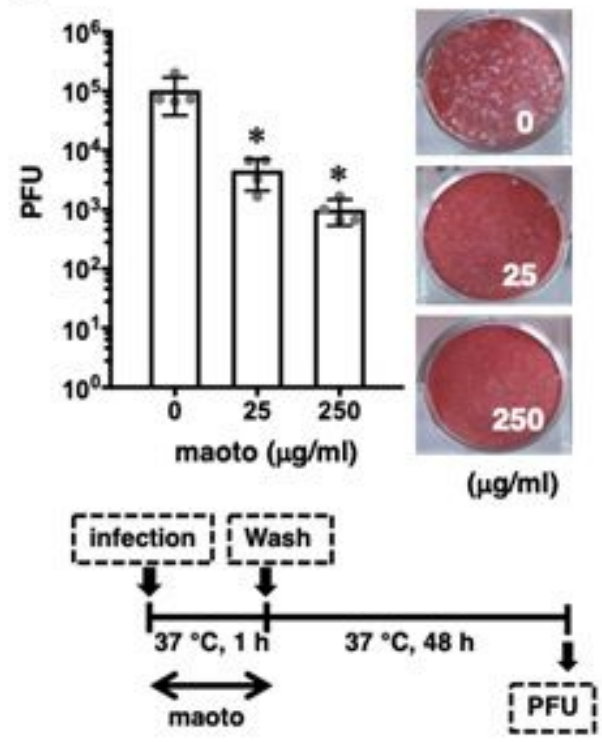

b
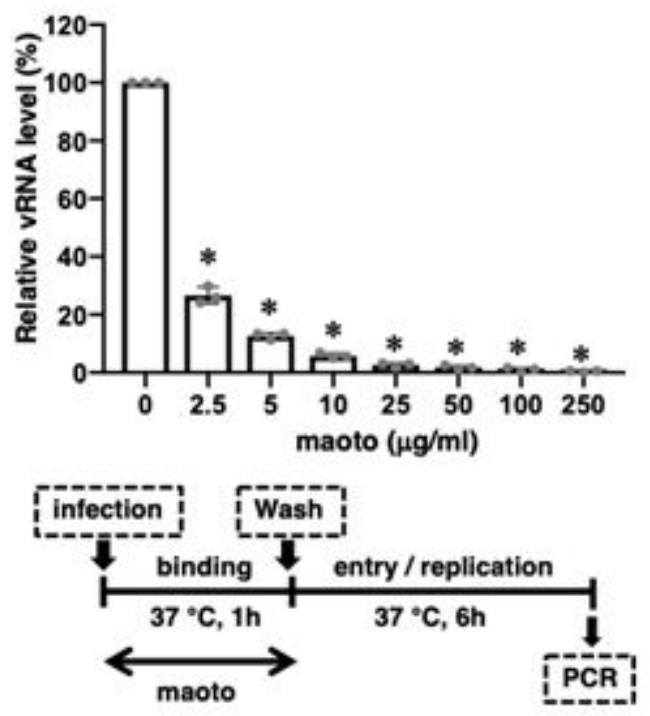

d
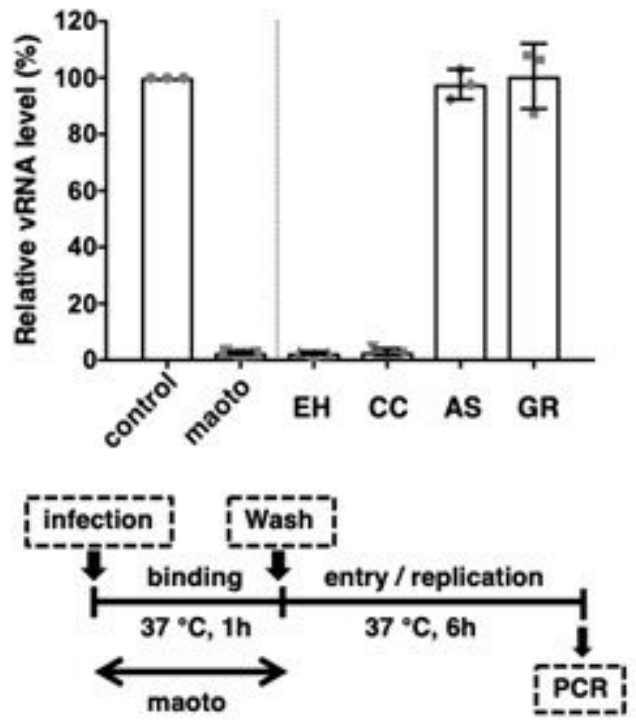

Figure 1

Anti-RSV activity of maeoto in cultured cells in the early phase of the viral life cycle 
a

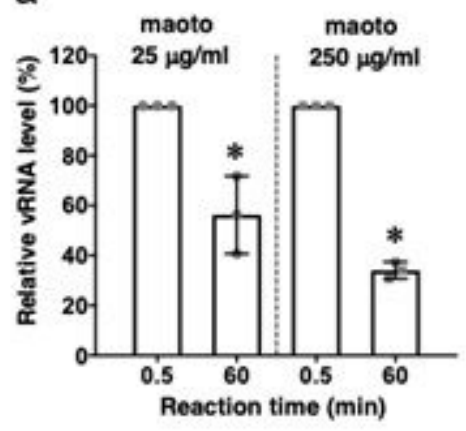

C

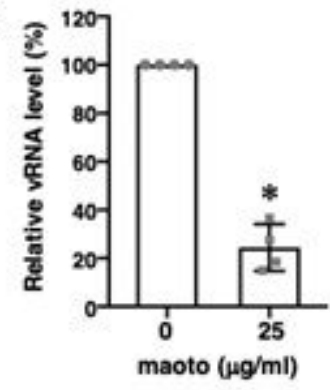

d

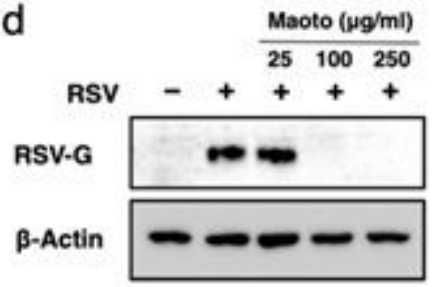

b

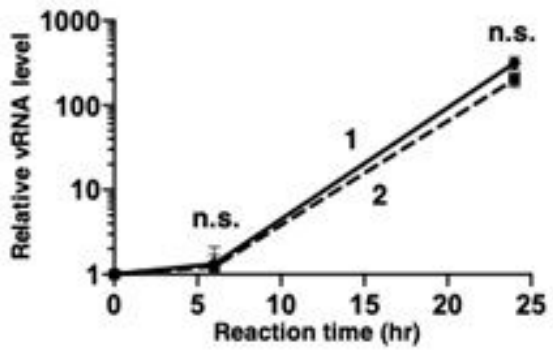

[intection [ [wash] [wash]

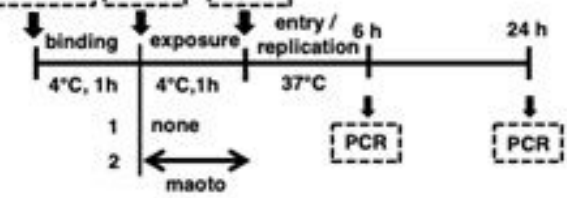

e
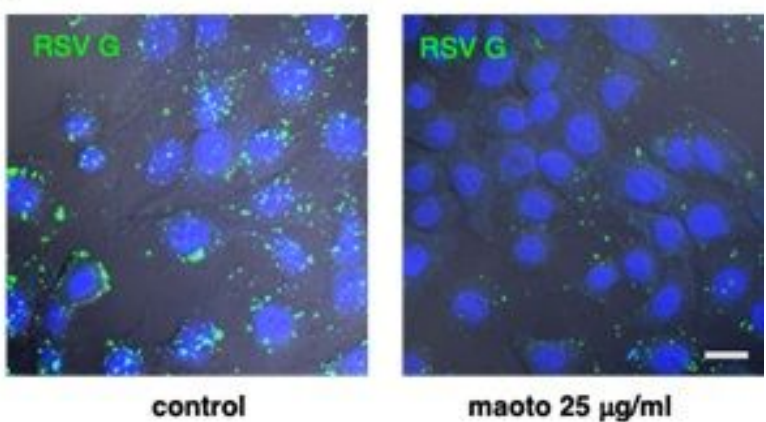

Figure 2

Direct inhibitory effect of maoto on RSV 
a

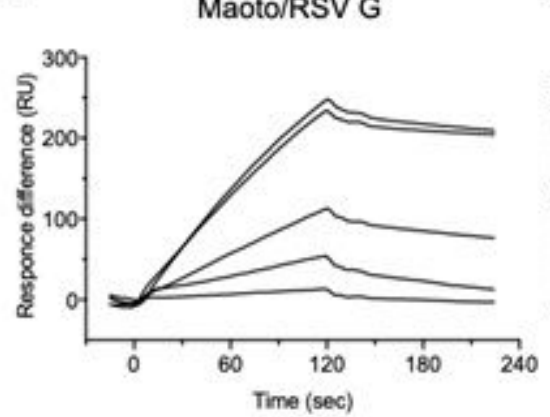

d

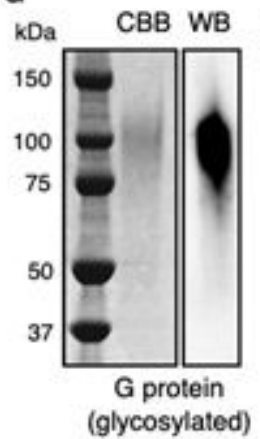

g

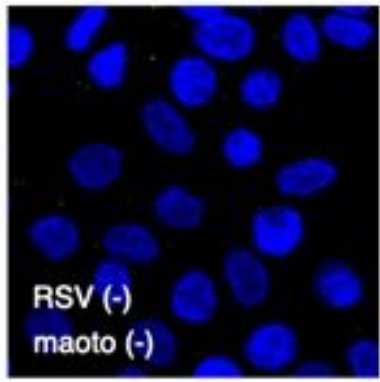

b
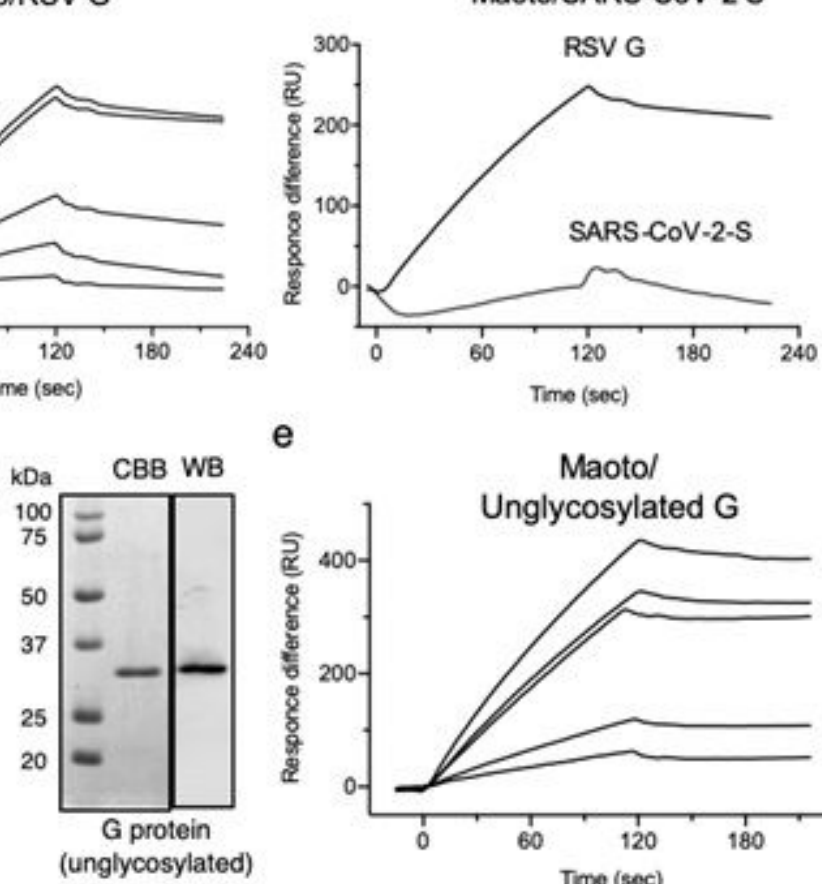

Maotol

e

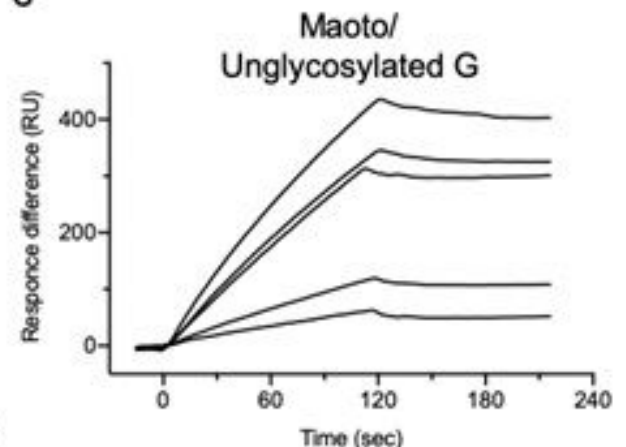

C

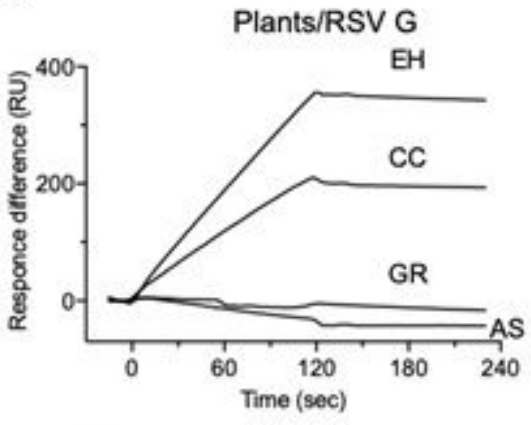

f

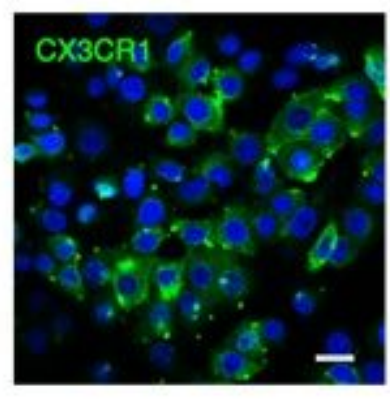

RSV ( + )

maoto $(-)$
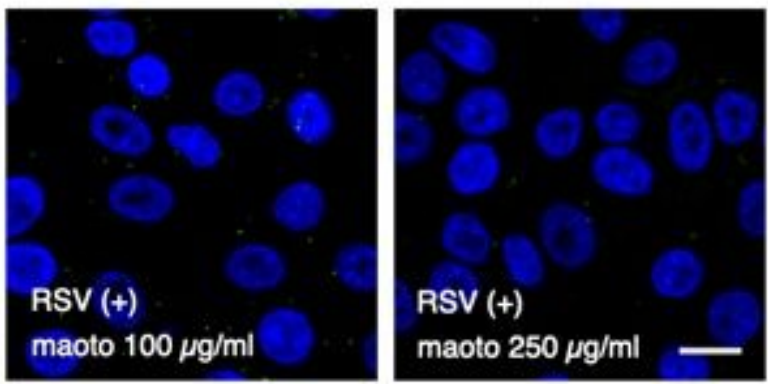

Figure 3

Specific binding of maoto to the central conserved domain (CCD) of RSV G glycoprotein 
a

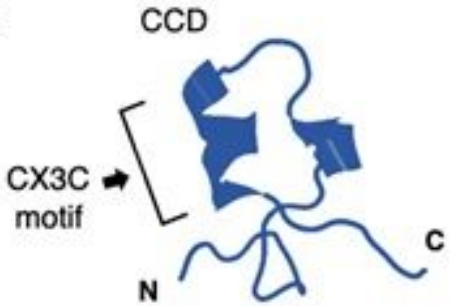

b

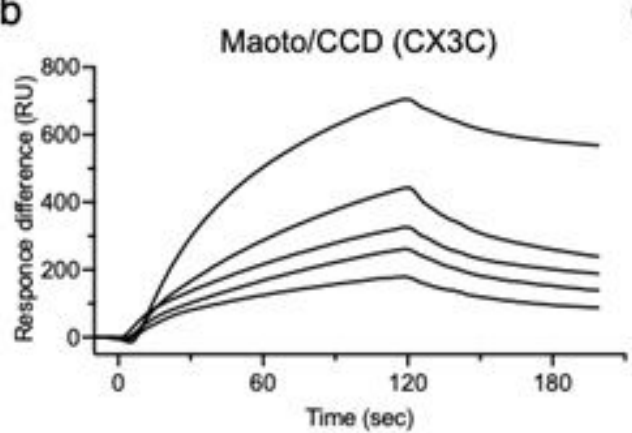

d

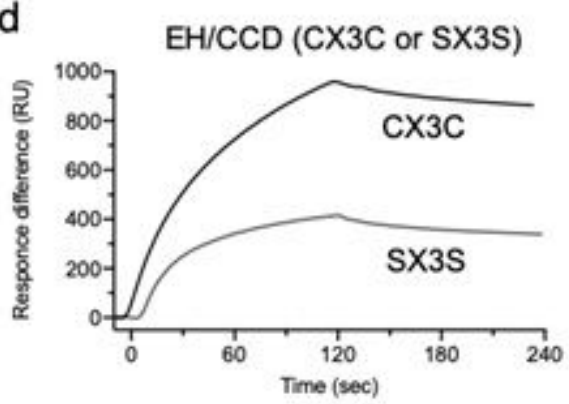

CX3C:

SX3S:
HFEVFNFVPCSICSNNPTCWAIC

HFEVFNFVPCSICSNNPTSWAIS

C
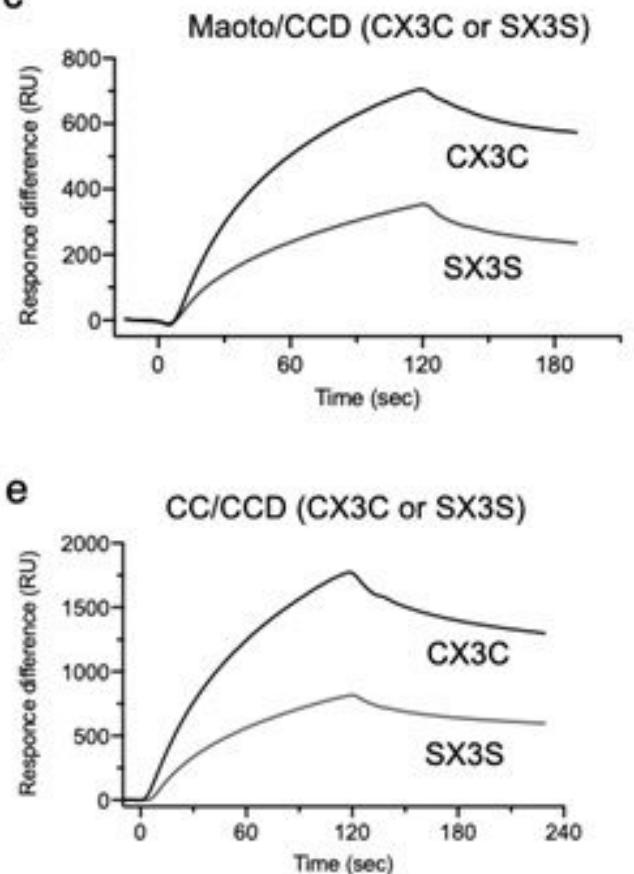

Figure 4

Specific binding of maoto to $\mathrm{CX} 3 \mathrm{C}$ on the RSV G protein CCD 
a

$$
\text { CX3CL1(fractalkine) }
$$

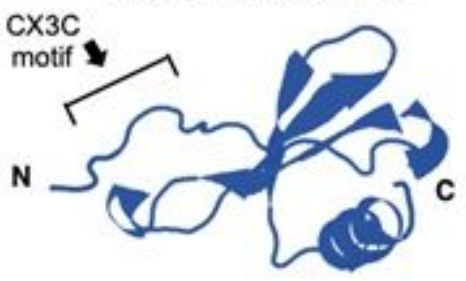

QHHGVTKCNITCSKMTSKIP

VALLIHYQQNQASCGKRAII

LETRQHRLFCADPKEQWVK

D AMQHLDRQAAALTRNG

b
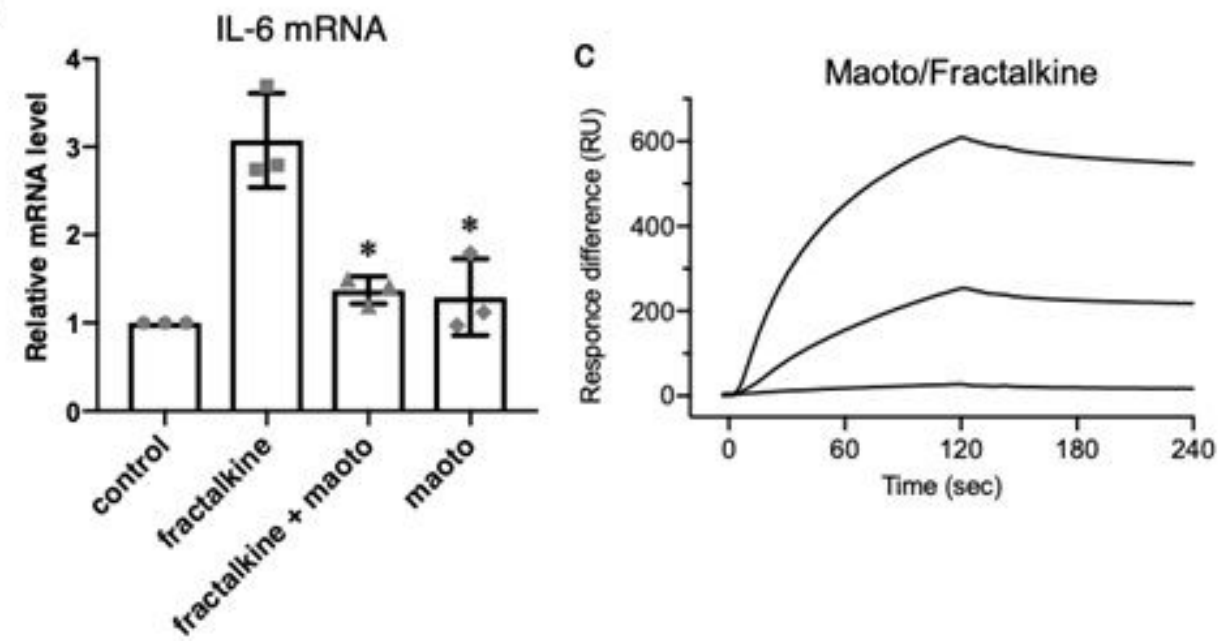

Figure 5

Interactions between maoto and the $\mathrm{CX} 3 \mathrm{C}$ motif

a

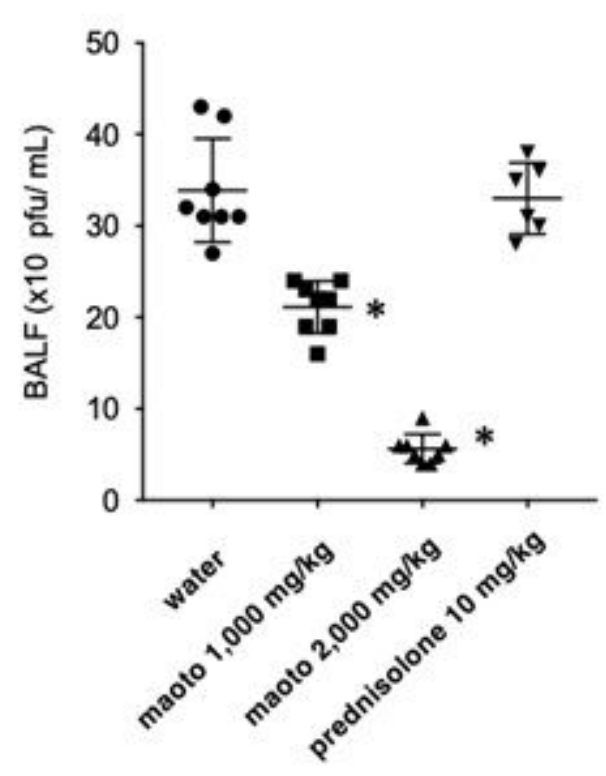

b
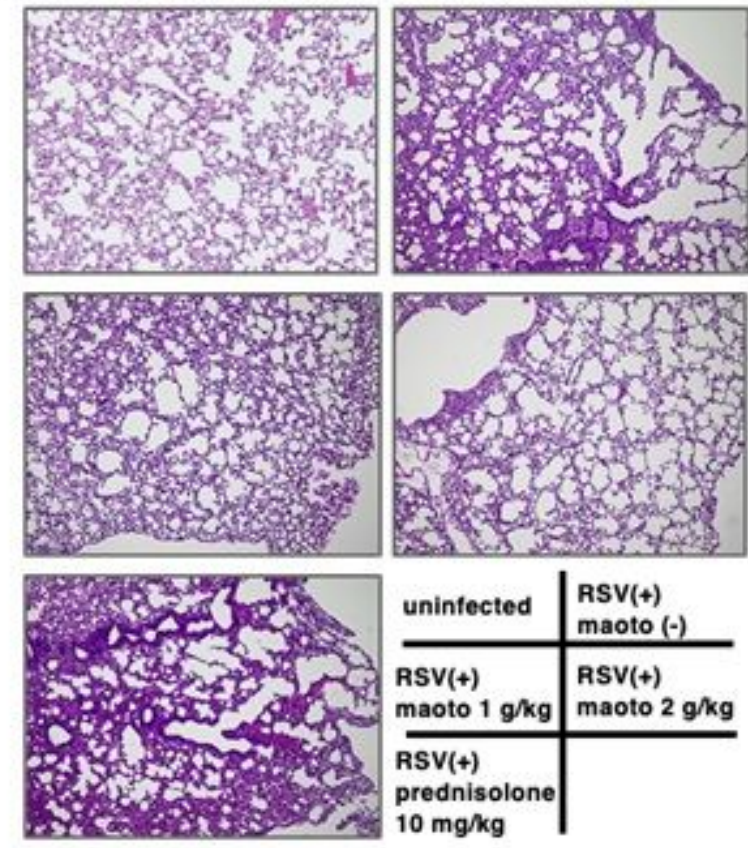

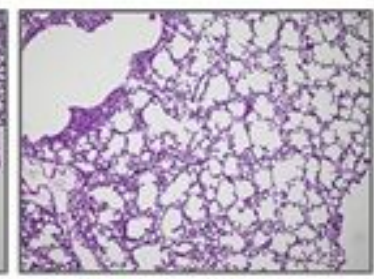

uninfected

RSV(t)

maoto $(-)$

RSV (t) $\quad$ RSV (t)

maoto $1 \mathrm{~g} / \mathrm{kg}$ maoto $2 \mathrm{~g} / \mathrm{kg}$

$\operatorname{RSV}(+)$

prednisolone

$10 \mathrm{mg} / \mathrm{kg}$

Figure 6 
Reduction of viral load by maoto in mice infected with RSV

\section{Supplementary Files}

This is a list of supplementary files associated with this preprint. Click to download.

- FullFigureCaptions.pdf

- SupplementaryMaterials.pdf 